\title{
Sistema de Manufactura Reconfigurable y Competitividad Industrial
}

Cesar H. Ortega Jiménez ${ }^{1}$

Ignacio Eguía Salinas ${ }^{2}$

\section{Resumen}

Este artículo ha decidido usar un modelo dinámico de competitividad de la Producción de Alto Rendimiento (High Performance Manufacturing, HPM), que presente las condiciones actuales en el escenario industrial a nivel mundial, para la futura implementación de Sistemas de Manufactura Reconfigurable (Reconfigurable Manufacturing Systems, RMSs). Así pues, aunque los RMSs pueden ser esenciales para la mejora continua, la globalización ha hecho difícil ganar ventaja (o aún paridad) competitiva a largo plazo con sólo implementar tales sistemas innovadores. Ello se debe en parte a la incesante e impredecible ( $y$ a veces predecible) evolución de las estructuras de mercado, de las demandas y de la tecnología de productos y de procesos. Por tanto, puede ser arriesgado el hacer predicciones de posibles rendimientos del impacto de futuros RMSs, desde mucha de la literatura teórica y empírica relacionada con el tema. Además, la evidencia de estudios empíricos en HPM muestra que la piedra angular de la competitividad industrial no es crear una nueva tendencia, sino más bien mejorar continuamente al seleccionar y adaptar, según sea necesario, sólo las practicas avanzadas de producción (PAPs) que se ajusten al contexto especifico de la fábrica, estableciendo vínculos entre las PAPs existentes y las nuevas por instalar. Así pues, tomando el contexto del HPM, se establecen las condiciones actuales para la implementación del RMS. Finalmente, este artículo hace sugerencias en relación al RM Se identifica posibles direcciones para futura investigación.

\section{Abstract}

This paper has decided to use a dynamic competitive model from High Performance Manufacturing (HPM), which presents current conditions in the global industrial stage, for the future implementation of Reconfigurable Manufacturing Systems (RMSs). Thus, although RMSs may be essential for continuous improvement, globalization has made it difficult to gain competitive advantage (or even parity) in the long run by just implementing such innovating systems. This is in part due to the incessant e unpredictable (and sometimes predictable) evolution of the market structures, from demands and from product and process technologies. Hence, it may be risky to make predictions from the possible performance impact of future RMSs, taking much of the theoretical and empirical literature related to the subject. Besides, evidence from HPM empirical studies show that the corner stone of the industrial competiveness is not to create a new trend, but to continually improve by selecting and adapting, according to needs, only the advanced manufacturing practices (AMPs) that better fit the specific context of the plant, establishing links among existing AMPs and new ones to be installed. Thus, taking the HPM context, this paper sets out current conditions for the implementation of RMS. Finally, this study make suggestions related to RMS and identifies possible directions for future research.

Palabras claves: Práctica Avanzada de Producción; Reconfigurable Manufacturing System; RMS; High Performance Manufacturing; HPM; contingencia; 


\section{INTRODUCCIÓN}

Esta época de rápido intercambio de información, y de benchmarking ha permitido que los académicos y profesionales de producción estén expuestos en todo momento a los últimos programas de producción,

Volumen I

$\mathbf{N}^{\circ} 2$

2010
Pág 98 tales como producción ajustada (lean manufacturing), justo a tiempo (just in time, JIT), calidad total, producción ágil (agile manufacturing), etc. Los pioneros de estos programas son normalmente organizaciones que han sobresalido internacionalmente, conocidos en la literatura como fabricantes de clase mundial (World Class, WC) o de alto rendimiento (High Performance, HP), y sus historias exitosas crean generalmente un tipo de tendencia, cuando otros fabricantes tratan de seguirles en sus subsecuentes intentos de implementar programas similares (la proposición de mejor práctica) (Schonberger, 1986, 2001; Schroeder y Flynn, 2001; Ortega, 2008).

Así pues, el sistema de manufactura reconfigurable (reconfigurable manufacturing system, RMS), cuya idea fue propuesta por primera vez hace más veinte años (Liles and Huff, 1990), no es todavía una de dichas historias debido a que la tecnología requerida se encuentra en distintas etapas de desarrollo. Actualmente, el RMS se está diseñando para que desde su puesta en marcha tenga la posibilidad del cambio rápido en estructura, así como en los componentes de hardware y software, para ajustar rápidamente la capacidad y funcionalidad de la producción dentro de una familia de una parte en respuesta a cambios repentinos o a requerimientos regulatorios (Koren et al., 1999). El diseño de dicho sistema parece ser muy prometedor, hasta el punto de ser uno de los lógicos pasos en la manufactura global. Sin embargo, antes de generalizar dicha frase, hay que tener algo de precaución, por lo que decimos a continuación.

Aun si fuera verdad de que todos los contextos de manufactura se han hecho efectivamente turbulentos e inciertos, debido a la capacidad de producción excesiva y la globalización económica, es muy improbable que todas las fábricas en todas las industrias se vean realmente forzadas a reexaminar sus programas de manufactura, cuando el RMS esté finalmente listo para el mercado (una vez que la herramienta de maquina reconfigurable/reconfigurable machice tool/RMT esté totalmente disponible), para que este nuevo sistema de tecnología pueda ser diseñado y operado eficientemente en este entorno siempre cambiante: puede no ser viable para todas las fábricas el sólo abandonar muchos de sus programas de manufactura para adoptar esta nueva iniciativa de manufactura.

Además, parece existir una controversia sobre la definición del RMS. Por un lado, la mayoría insiste en que el RMS es una práctica tecnológica intermedia entre la producción en masa y el sistema de manufactura flexible (flexible manufacturing system, FMS) (ej. Koren et al., 1999; Bi et al., 2007). Por otro lado, otros discuten que el RMS es una iniciativa de manufactura, que encierra a la producción ajustada (un enfoque más holístico) (ej. Mehrabi et al. 2000b). Finalmente, la literatura parece sugerir algunas veces que el RMS es tan sólo una nueva máquina (ej. Mehrabi et al. 2000a). 
A pesar de dichas confusiones y controversias, debido a que el interés en el RMS y su efecto sobre el rendimiento competitivo han crecido, ha habido una respectiva proliferación de investigación. Sin embargo, todo el trabajo sobre el RMS parece ser caracterizado por tener un enfoque limitado, especialmente en relación a verlo principalmente como un recurso físico competitivo. Asimismo, parece que todavía no existe un fundamento teórico, ni mucho menos uno empírico, para la proposición de que el RMS tiene un valor competitivo como parte de una estructura sistémica dentro de las fábricas productivas. Los estudios ponen poca o ninguna atención tanto a las contingencias (contexto), como a las interconexiones (ambas explicadas más abajo) involucradas en adoptar e implementar un RMS. Casi la totalidad de la literatura no presta suficiente atención a la amplia naturaleza multidimensional del rendimiento de la fábrica.

Cuando se toman en consideración todos estos importantes tópicos de investigación, y aún si las contingencias de la fábrica llevan al RMS, se deben considerar asuntos empíricos importantes al implementar un nuevo programa de manufactura. Por ejemplo, el RMS debe ser unido a las prácticas de producción presentes en una fábrica. Por ello, el RMS no puede ser un fin en sí mismo, debido a que su trayectoria al alto rendimiento no sólo depende de la contingencia de la fábrica, sino también de la interconexión con otras prácticas y áreas de la fábrica. Ambas, la contingencia y la interconexión entre prácticas, son los fundamentos claves de la perspectiva de la Producción de Alto Rendimiento (High Performance Manufacturing, HPM), que consiste en un conjunto integrado de procesos diseñados para alcanzar una ventaja competitiva mundial sostenible por medio de la mejora continua de la capacidad de fabricación (Schroeder and Flynn, 2001). Así pues, tomando el RMS, en conjunto con HPM, este artículo se hace la siguiente pregunta de investigación: ¿existen condiciones actuales en el contexto HPM para la implantación futura del RMS? Dicha pregunta se contesta por medio de un estudio exploratorio, donde se busca estructurar e identificar puntos comunes entre el RMS y la competitividad industrial.

Así pues, usando una perspectiva HPM, esta investigación conduce una revisión crítica de la posible relación del RMS con algunas de las iniciativas del HPM implementadas actualmente (tales como la producción ajustada y el FMS), como una medida de comparación inicial para un potencial escenario de implantación del RMS. Así pues, el epígrafe 2 revisa brevemente el HPM y algunos de sus programas existentes, para examinar globalmente las condiciones presentes de contingencia e integración entre prácticas de producción escenificadas para el RMS. En la sección 3, se hace una primera aproximación del RMS en el escenario del HPM. El punto de inicio es la conceptualización del RMS, donde Koren et al. (1999) la definen en línea con sistemas tal como el FMS. Por tanto, ya que el FMS es parte de la automatización flexible ${ }^{1}$ (Filippini et al., 1998; 2001), esta parte explicará el programa de tecnología de manufactura, del cual es parte la automatización flexible. En la última sección (4) se presenta un resumen con algunas de las direcciones posibles de investigación futura identificadas.

Sistema de Manufactura Reconfigurable y Competitividad Industrial 


\section{REVISIÓN DE LA LITERATURA}

Desde que el término "Fabricación de Clase Mundial/World Class Manufacturing (WCM)" fue introducido por Hayes and Wheelwright (1984), un tema central en la dirección/gestión de producción y operaciones

Volumen I

$\mathbf{N}^{\circ} 2$

2010 (DGPO) ha sido mejorar la fabricación y diseñar para alcanzar estándares de clase mundial (alto rendimiento). Dichos autores usaron el término WCM para referirse a las capacidades de los fabricantes japoneses, estadounidenses y alemanes para competir en mercados mundiales. El concepto fue consecuentemente popularizado por Schonberger (1986). En sus inicios, se refería como WCM a las compañías que habían alcanzado el mayor nivel de rendimiento en una o más áreas estratégicamente importantes, al adoptar procesos establecidos para mejorar cómo se gestionaban las operaciones (Hayes and Wheelwright, 1984; Voss and Blackmon, 1996; Flynn et al., 1997). Estos procesos están constantemente variando debido a los cambios globales que se llevan en contextos empresariales. Esta activa operación es una de las razones por las que la conceptualización de WCM tenga un marco de referencia dinámico y abierto.

La literatura más reciente de WCM ha sido expandida a una forma empírica. Por ejemplo, Schonberger (2001) un libro acerca de sus resultados empíricos en EEUU. Muchos otros estudios empíricos fueron hechos como parte de un proyecto internacional de investigación sobre la Producción de Alto Rendimiento (High Performance Manufacturing, HPM). Los primeros 150 o más artículos y resultados de este proyecto han sido publicados en un libro titulado "High Performance Manufacturing: Global Perspectives", editado por Schroeder and Flynn in 2001. Este libro explica que los métodos de WCM/HPM no son de propósito general, sino que deben ser adaptados a la industria y el entorno de cada fábrica. También encuentra que los caminos para lograr HPM pueden ser diferentes en Alemania, Gran Bretaña, Italia, Japón y Estados Unidos.

Así pues, una contribución importante del proyecto HPM es la noción del enfoque de contingencia, que va más allá de sólo imitar las mejores prácticas de los competidores internacionales (Ketokivi and Schroeder, 2004). El enfoque de contingencia aduce que las prácticas avanzadas de alto rendimiento deben ser adaptadas y ajustadas a las fábricas en sus situaciones particulares. Otra prestación distintiva del HPH es el énfasis que se le da al vínculo entre las prácticas avanzadas de producción (PAPs). Los resultados empíricos muestran que vincular una PAPs con otra es lo que lleva al HPM, y ésta es una necesidad continua que debe ser renovada constantemente. Así pues, no es que el éxito sigue a las fábricas sólo por el número de PAPs que son implementadas o de las últimas PAPs, sino de cómo las PAPs se relacionan entre sí y cómo se erigen acumulativamente unas sobre otras. El vínculo de PAPs provee la base para unir nuevos programas en la dirección de la fábrica. Otro mensaje importante del HPM es que WCM en sí mismo es un blanco elusivo y con un nivel elevado de variación en el rendimiento. En conclusión, es necesario que una fábrica diagnostique cuidadosamente su situación y sólo entonces decida su camino deliberado de mejora continua. Esto, por supuesto, es más fácil de decir que de hacer, que lleva a una explicación de por qué HPM es tan difícil de lograr y sostener en la realidad. 
Con lo anterior, se puede actualizar el concepto inicial de WCM al de HPM, diciendo que es un grupo integrado de procesos diseñados para alcanzar ventaja competitiva mundial sostenible por medio de la mejora continua de la capacidad de manufactura (Schroeder and Flynn, 2001). Siendo un concepto dinámico, el HPM examina a cada momento las iniciativas de manufactura para su posible inclusión como parte de los procesos de producción, dependiendo de la contingencia de la fábrica (contexto) y de la posible integración de la nueva iniciativa con lo que la fábrica ya está haciendo o va hacer. Esta perspectiva dinámica permite que su modelo en especial sea amplio y siempre cambiante, como puede verse en la siguiente sección.

Antes de continuar, es necesario definir el significado de iniciativa de manufactura (también conocida como área o programa de prácticas de manufactura). En este estudio se considera a la iniciativa de manufactura como una acción innovadora que modifica las prácticas de gestión y los sistemas tecnológicos y organizacionales de una fábrica con el objeto de mejorar la competitividad, por mayores rendimientos medidos multidimensionalmente por el tiempo (velocidad), el servicio, la calidad, la fiabilidad (entrega), el costo, etc. No existe una única definición de "iniciativa" en la literatura de la DGPO, ya que algunas veces se le denomina como "mejor práctica", otras como "innovación". Sin embargo, y pesar de la variedad de términos usados, las iniciativas de manufactura muestran una considerable homogeneidad, hasta el punto de que puedan ser categorizados en tres tipos de tipologías (Filippini et al., 1998): 1) iniciativas de carácter primordialmente tecnológico (ej. CAD, FMS, RMS, etc.); 2 ) iniciativas de carácter mayoritariamente organizacional (ej. RR.HH., relaciones con proveedores, calidad total, etc.); y 3) iniciativas de carácter tanto tecnológico como organizacional (ej. manufactura ajustada, manufactura ágil, ingeniería concurrente, JIT, etc.)

Finalmente, las fábricas pueden lidiar con el entorno cambiante de los mercados por medio de la implementación de las iniciativas de manufactura, también conocidas como prácticas avanzadas de producción (PAPs). Los conceptos de producción dentro de estas iniciativas tienen en común el hecho de que interactúan simultáneamente con varias funciones de las fábricas, produciendo cambios significativos. En última instancia, la meta es mejorar el rendimiento general. Para ello, existen un gran número de iniciativas de manufactura documentadas en la literatura que apuntan para tal mejora competitiva. Es responsabilidad de la gerencia escoger e implementar los conceptos que potencialmente pueden alcanzar los objetivos de manufactura. Muchas de estas iniciativas de manufactura pueden componerse de grupos de prácticas interrelacionadas e internamente consistentes, haciendo un área o programa de prácticas, que puede llamarse programa de manufactura (por ejemplo, el programa de RR.HH. tiene iniciativas/prácticas tales como cooperación, capacitación, rotación, etc.). Además, algunas de estas iniciativas pueden caracterizarse como un subconjunto de otro $u$ otros programas de manufactura (ej. FMS como parte de la tecnología de procesos). Dependiendo de los requerimientos particulares de la fábrica, hay diferentes enfoques multidimensionales disponibles que reúnen una gran variedad de estas prácticas de manufactura, que a su vez pueden ser de varios programas en sistemas integrados tales como producción ajustada, personalización masiva, producción ágil, etc. Estos sistemas pueden distinguirse entre sí de varias formas como, por ejemplo, el número de iniciativas que realizan, una tendencia hacia ciertos tipos de iniciativas (ej. duras/tecnológicas, blandas/organizacionales, o combinadas). Así pues, tomando el concepto del HPM, se exponen a continuación algunos escenarios actuales de las PAPs para el RMS. 


\section{RMS PARA EL HPM}

Históricamente, la idea con la que la mayoría de las empresas están familiarizadas es recomendar a los gerentes de producción a que adopten todas las iniciativas de producción que aparecen como tendencias. Este estudio, por el contrario, se desmarca de tal idea, al asociar a la empresa el concepto cuyo enfoque es vincular sólo las prácticas de producción (con adaptaciones) que conjuntamente lleven a la clase mundial. Pero antes de tal vinculación entre las prácticas, debe haber un plan estratégico de contingencia, basado en la situación particular de la empresa, para seleccionar, adaptar (cuando sea necesario) e implementar las prácticas, o el esfuerzo de diseño/implementación no tendrá el efecto deseado (un negocio más exitoso). Este proceso de contingencia y de vinculación debe estar unido con una trayectoria deliberada de mejora continua. Dicho enfoque, conocido como producción de alto rendimiento o High Performance Manufacturing $(\mathrm{HPM})^{2}$, será usado consecuentemente para estudiar las condiciones actuales puestas en escena para la implementación futura del RMS.

\subsection{RMS: más allá de FMS y de LEAN}

La búsqueda para desarrollar la tecnología para los sistemas de manufactura reconfigurable (RMS) empezó a mediados de los noventas como una respuesta costo eficaz a las demandas del mercado para personalización y grado de reacción. De acuerdo a Koren et al. (1999), RMS está siendo diseñado para el cambio rápido en estructura, que incluye tanto los componentes de hardware como los de software, para ajustar rápidamente la capacidad y funcionalidad de la producción, dentro de una parte de la familia de un producto, en respuesta a cambios repentinos en el mercado. Koren y sus colegas explican que para que un sistema de producción sea realmente reconfigurable, debe poseer ciertas características, que por lo menos incluyen: 1) modularidad de diseños de componentes; 2) integrabilidad para tanto una integración instantánea como para la futura integración de nuevas tecnologías; 3) convertibilidad para permitir una rápida transición entre productos y adaptabilidad rápida de sistemas para productos futuros; 4) diagnosticabilidad para identificar velozmente las fuentes de problemas de calidad y de fiabilidad; 5) personalización para ajustar la capacidad y flexibilidad diseñada del sistema a las aplicaciones; y 6) la escalabilidad para cambiar rápida y económicamente la capacidad de forma incremental Por un lado, NGM (1997) ha dicho que los RMS necesitarán herramientas nuevas y eficaces para adaptarse posiblemente a cambios frecuentes, a la introducción de nuevos productos, y a corridas cortas de producción sin perjudicar seriamente la producción. Así pues, la motivación para introducir RMS se basa en la expectativa de obtener algún beneficio económico al incrementar la reusabilidad y reducir el exceso de capacidad y/o de funcionalidad presente en otros tipos de sistemas de manufactura (El Maraghy, 2006).

Por otro lado, varios estudios (ej. NRC, 1998; Pham et al., 2004) han señalado que, para mantenerse competitivo, las empresas deben poseer tecnología de ingeniería reconfigurable, ya que futuros RMS prometen ser costo-eficaces y de rápida respuesta a "todos" los cambios del mercado. Además, aunque el RMS todavía no está totalmente operativo (menos aún implementado en alguna empresa), la literatura especializada (ej. Mehrabi et al., 2000b) parece indicar que, tan pronto el RMS se haga disponible, seguramente se considerará como un programa de "mejores prácticas". De hecho, proponentes de este enfoque (ej. Koren et al., 1999) creen que el RMS tiene el potencial para ofrecer una mayor solución económica que los actuales sistemas de automatización flexible, tales como el FMS, al incrementar la vida y utilidad de un sistema de producción. Algunos autores (ver Stecke, 2005), aún van más allá al predecir también más flexibilidad en el RMS.

2 Conocido también como World Class Manufacturing (WCM) 
Se podría seguir hablando acerca de los potenciales de tecnologías reconfigurables, pero hay que tener cautela antes de ponerle etiquetas al RMS como la iniciativa o la tecnología de producción más moderna y segura para lograr alto rendimiento en el futuro cercano, aun si es el objeto de grandes esfuerzos de investigación de su desarrollo alrededor del mundo. A pesar de la tecnología pueda estar disponible hoy para lograr un RMS útil y asequible, su argumento costo-eficaz todavía necesita verificarse. En términos prácticos, esto quiere decir que, considerando el ciclo de vida total de necesitado en todo el sistema, RMS es más costo-eficaz, de más rápida reacción en el tiempo que la presente tecnología de automatización flexible (ver Amico et al., 2003). Asimismo, existen al menos 15 desafíos prácticos y fundamentales que permanecen como preguntas abiertas, que EIMaraghy (2006) enumera como áreas de investigación para completar el desarrollo del RMS.

Es más, inclusive después que el RMS esté totalmente operativo (y dando las prestaciones que promete), aún queda una pregunta fundamental que contestar: ¿es RMS una práctica universal para todo tipo de fábrica? El argumento de contingencia del HPM, mencionado en la sección 1, tiene algo que responder acerca de esta pregunta: depende de la fábrica. Por supuesto, esto no debería ser una excusa para hacer nada. Así pues, como la competición económica global y los cambios rápidos sociales y tecnológicos han forzado a la industria en general a enfrentar un grado de reacción por parte de la producción, ¿qué es lo que los productores de clase mundial (HPM) están haciendo ahora para reunir los requisitos de rendimiento del grado de reacción con las prácticas de producción disponibles? Están todas las fábricas listas para el RMS? Estas preguntas servirán para enriquecer la pregunta de investigación, formulada en la introducción, cómo guía para el resto de esta sección y del artículo.

Incluso si todas las industrias experimentaran entornos siempre cambiantes, es poco probable que, en el corto plazo, todas las fábricas se vean forzadas a reexaminar sus programas de producción, de modo que un nuevo sistema de tecnología como el RMS pueda ser diseñado y operado eficientemente. En otras palabras, no es factible para todas las fábricas simplemente abandonar muchos de sus programas de producción para adoptar esta nueva iniciativa. Por otro lado, como este artículo ha señalado, parece haber una controversia por resolver acerca del concepto funcional del RMS.

Al mismo tiempo, existen otros asuntos claves que hay que considerar al implementar un nuevo programa de producción. Por ejemplo, Cua et al. (2006) Evalúan que cada cinco a diez años se introduce un nuevo programa, tal como lean, calidad total, FMS, etc., como la panacea para lograr competitividad; e incluso cuando algunos de estos programas fallan en la práctica por ciertas fábricas, las dos causas principales dadas por los académicos y profesionales son la implementación parcial y la incompatibilidad de sistemas en la fábrica. Dado que la mayoría de la investigación considera dichos programas primordialmente de forma aislada, Cua y sus colegas han propuesto que también hay que considerar el vínculo entre dichos programas al implementar prácticas comunes a todos los programas existentes y vincular nuevos programas con las prácticas actuales.

Por tanto, como se dijo antes (p. 2), la tecnología reconfigurable no puede ser un fin en sí misma, ya que debe vincularse a otras prácticas y áreas de la fábrica en el camino hacia la competitividad. En principio, la persecución de un mejor rendimiento y de ventaja competitiva obliga a que las fábricas no sólo obtengan el último equipamiento, sino también que desarrollen recursos y capacidades que no pueden ser fácilmente duplicadas, y por los cuales no existen sustitutos disponibles. De un estudio anterior, Schroeder et al. (2002) han concluido que, en el contexto de la perspectiva basado en el recurso de la fábrica, el HPM es más probables del: 1 ) aprendizaje interno tal como la capacitación inter- 
disciplinaria y los sistemas de sugerencias; 2) aprendizaje externo tal como clientes y proveedores; y 3 ) procesos y equipos patentados.

Todos ellos desarrollados por la fábrica para formar tales recursos y capacidades. Dos implicaciones de los resultados empíricos de Schroeder y sus colegas son que los recursos tales como el equipo estándar y em-

Volumen I

$\mathbf{N}^{\circ} 2$

2010
Pág 104 pleados con habilidades genéricas obtenibles en mercados con factores de producción no son tan eficaces en lograr altos niveles de competitividad/rendimiento para la fábrica, por estar libremente disponibles a los competidores, y que el aprendizaje interno y externo juegan un papel importante en desarrollar recursos que son imperfectamente imitables y difíciles de duplicar.

Así pues, tomando algunos de los programas de producción, este articulo explora la literatura de HPM para examinar globalmente las condiciones presentes de la contingencia y vínculos de prácticas en fábricas en el escenario mundial para la reconfigurabilidad. El punto de partida para ello es la conceptualización misma del RMS con dos de sus temas claves: 1) Varios autores (ej. Koren et al., 1999) han formulado al RMS como un sistema que revoluciona o, por lo menos, evoluciona del FMS, y como tal se han incluido algunos de sus estudios empíricos como parte de HPM; y 2) La literatura del RMS va más allá, al decir explícitamente que este nuevo sistema tiene los medios de mejorar multidimensionalmente al rendimiento de no sólo el FMS, sino también de ambas, la producción ajustada (lean)y la producción en masa (Mehrabi et al., 2000b; Bi et al., 2007). Dado que la producción lean engloba muchos de los programas HPM tales como JIT, RR.HH., calidad total, mantenimiento productivo total, etc., el presente artículo considera ello como otro asunto clave a tratar.

La automatización flexible, como primera clave, es un intento de combinar las ventajas de la automatización fija con las ofrecidas por la automatización programada. Usando dicho método, las fábricas pueden obtener simultáneamente bajos costos unitarios y un alto grado de flexibilidad. La automatización flexible se define como un sistema integrado de hardware y software que hace posible diseñar y producir automáticamente una variedad predefinida de productos. Además del FMS, existen varios tipos de automatización flexible, tales como almacenamiento y transporte automatizado, células de producción y producción numérica, producción por control numérico por computadora (CNC)/control numérico directo (DNC), etc. Debido a las características del RMS, se puede considerar el siguiente paso del FMS, y como tal el RMS debe considerarse también como parte de la automatización flexible.

De acuerdo a Goldman et al (1995), la producción lean es una filosofía de gestión que se enfoca en:1) la obtención de un producto bien en la primera vez; 2) esfuerzos de mejora continua; 3 ) la calidad de productos y procesos; y 4) minimizar el desperdicio por medio de diferentes fuentes tales como transportación (movimiento innecesario de materiales), inventarios (exceso de inventario no requerido directamente para las ordenes presentes), movimientos (pasos extras tomados por empleados por la ineficiencia del diseño), tiempos de espera (periodos de inactividad), sobre-producción (ocurre cuando la producción debió haber parado), sobre-procesamiento (reelaborar y reprocesamiento), productos defectivos (no se conforman a las especificaciones o expectaciones).

Por la naturaleza tecnológica del RMS, la siguiente sección explica el programa de la tecnología de producción, del cual es parte la automatización flexible. Después, la sub-epígrafe 3.3 muestra un primer enfoque de la relación entre la producción lean y el RMS. 


\subsection{Tecnología de producción y el RMS}

Actualmente, la siguiente generación de sistemas de manufactura (next generation manufacturing systems, NGMS), que se espera estén mejor equipadas para sobrevivir los nuevos entornos competitivos, está inundando a la producción con futuras modas, tales como sistemas de manufactura holonica (holonic manufacturing systems, HMS), compañías fractales, sistemas de manufactura autónoma y distribuida (autonomous and distributed manufacturing systems, ADMS), sistemas de coexistencia humano-máquina (human-machine coexistence systems, HUMACS), factores sensoriales humanos para ciclos de vida total del producto (human sensory factors for total product life cycle, HUTOP), sistemas de manufactura bilógica (biological manufacturing systems, BMS), etc. En relación a la flexibilidad, dichas futuras modas están llevando hacia la reconfigurabilidad en sistemas, que están siendo desarrollados, tales como el sistema de manufactura flexible escalable (scalable flexible manufacturing systems, SFMS) y el sistema de manufactura reconfigurable (reconfigurable manufacturing systems, RMS). El enfoque del presente estudio es el RMS.

La literatura actual de reconfigurabilidad cita al cambio tecnológico hacia el RMS como un prerrequisito para la competitividad y supervivencia de las empresas, y aún economías enteras (Mehrabi et al., $2000 b)$. Sin embargo, ¿será el rendimiento tecnológico, como el que RMS promete, realmente un factor crítico para el éxito de la producción? La respuesta, a primera vista, parecería que se sí, debido a que la implementación eficaz y el uso de tecnología se citan como armas estratégicas en las batallas de una empresa contra la competencia, con tanta frecuencia que todo el mundo cree en ello. Pero al dejar la superficie e ir más profundo dentro del tema, aparecen mucho asuntos acerca de lograr competitividad/alto rendimiento, tal como las implicaciones en la DGPO cuando se implementan y usan programas eficaces y extraordinarios de tecnología con sus diferentes prácticas y dimensiones (Schroeder and Filin, 2001).

Como se explicó en el sub-epígrafe anterior, desde el punto de vista de tecnología (FMS), este artículo considera que el RMS se ajusta mejor como parte de automatización flexible, que pertenece a la tecnología de procesos, y este último es una de las partes más importantes del programa de tecnología.

Por su lado, la tecnología de procesos puede definirse como el equipamiento y los procesos para fabricar productos. La literatura del HPM (ej. Maier, 1997; Ortega, 2009) explica algunas de las dimensiones claves (que se pueden ligar a una posible implementación del RMS) de cómo la tecnología de procesos puede influenciar al rendimiento: 1) el uso eficaz de la tecnología puede lograr la flexibilidad para cambios en el volumen de la producción, en el programa de manufactura, y en el tipo de producto a ser fabricado; 2) la aplicación de sistemas detallados de la calidad de gestión en conjunto con la tecnología usada en la producción resulta en productos de alta calidad (ej. énfasis en máquinas que operan sin incidentes y con baja desviación de tolerancias, de residuos, y de reelaboración, así como el uso de máquinas con inspecciones automatizadas); 3) la tecnología de procesos influye los bajos costos (ej. economías de escala, así como economías de gama, bajos tiempos muertos de equipos causados por los paros de producción, por los tiempos cortos de preparación, y por los bajos porcentajes de reelaboración y residuos); 4) la tecnología de procesos puede ayudar a asegurar a la capacidad de las fábricas de cumplir con las demandas de los clientes (en relación a la entrega a tiempo y los tiempos cortos de entrega de la producción).

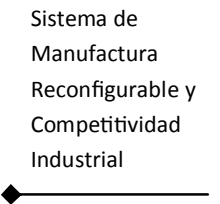


Para hacer una gran diferencia en lograr HPM en estas dimensiones, la tecnología de procesos debe incluir no sólo aspectos duros tal como la automatización flexible (FMS), sino también de aspectos suaves tal como trabajar con proveedores para desarrollar nuevas tecnologías y cooperación inter-funcional en la empresa (Maier and Schroeder, 2001).

Volumen I

$\mathbf{N}^{\circ} 2$

2010
Pág 106
Más allá de estas importantes dimensiones, según el HPM (Maier and Schroeder, 2001), el programa de tecnología comprende aspectos de no sólo los procesos, sino también de productos (tecnología de productos), así como de sistemas de la información (tecnología de la información y comunicaciones, TIC). Estos diferentes aspectos están vinculados entre sí en el sistema de producción de una fábrica. Por tanto, se asume que las tres dimensiones o aspectos son factores fundamentales con impactos directos e indirectos a la competitividad y el rendimiento de la fábrica. Además, el uso eficaz de la tecnología misma está influenciado por otros muchos factores.

Incluso con esta perspectiva detallada, en términos de competitividad, es aún insuficiente con sólo ser el líder tecnológico en determinada industria. En el HPM, el sistema tecnológico de una fábrica no es una isla debido, a que las tres facetas/aspectos de tecnología deben ajustarse a la fábrica, donde tienen que estar incrustados e interactuando. Así pues, la tecnología apoya los procesos en la fábrica, y las estructuras de este último apoyan la tecnología en todos los niveles de la fábrica (ej. productos, procesos de producción, TIC, RR.HH., etc.) tienen que ajustarse entre sí. Además, la eficacia de las prácticas del HPM están interrelacionadas con la tecnología, e influencia el éxito del sistema tecnológico de una fábrica: la tecnología y otros programas de prácticas del HPM influencian juntos la competitividad y el rendimiento. La falta de vinculación entre la tecnología y otras áreas de la fábrica puede ser una importante causa de fracaso (Maier and Schroeder, 2001).

Lo que la fábrica haga (y hasta lo que no haga) se puede ver reflejado en sus resultados. Por tanto, la decisión de usar ciertas prácticas de tecnología, u otras, o absolutamente ninguna (ninguna acción tomada) tiene siempre un impacto sobre el rendimiento. Ello deja espacio para diferencias que pueden distinguir a los productores de alto rendimiento (HP) de los productores estándares. Por ejemplo, si se considera las diferentes tecnologías que están en uso, los HP son más innovadores y, por tanto, tienen mayor posibilidad de que los productores estándares puedan introducir innovaciones tales como CAD, CNC/DNC, FMS, o el aún por estrenar RMS.

Por su lado, el proyecto HPM (ej. Maier and Schroeder, 2001) también sugiere la importancia de algunas prácticas específicas de tecnología, que mejoran la competitividad del proceso de la tecnología tales como la implementación eficaz de nuevos procesos, la consideración de la capacidad productiva en la fase de diseño del desarrollo de nuevos productos, la simplicidad en el diseño del producto, la habilidad de la fábrica para anticipar nuevas tecnologías, y el procesos eficaz.

Sin embargo, estas prácticas, o incluso cualquier práctica de producción, no funcionarán sin el escenario apropiado en la fábrica. Así pues, el equilibrio de esfuerzos es más importante que ser fuerte en la mayoría de las prácticas, mientras se falla en algunas de las prácticas que sirven de sustento. En principio, la baja innovación del producto (tecnología de productos) va en línea con la baja innovación en el equipo de producción (tecnología de procesos). En relación a la competitividad/alto rendimiento, el proyecto HPM ha concluido que efectivamente tanto la tecnología de procesos como la de productos juegan muy bien juntas por medio de una relación balanceada que se encuentra entre los productores HP (Schroeder y Flint, 2001). 
En este camino de producción para que las fábricas logren y mantengan el alto rendimiento y la competitividad, la idea de balance también se ve apoyada por lo reciente del equipo (tecnología de procesos) y el porcentaje de ventas de productos introducidos (tecnología de productos). Mientras que los productores HP parecen estar usando un porcentaje significativamente mayor del equipo de producción, uno debe tener el cuidado de no concluir que la inversión en el equipo futuro tal como la RMT mejorará automáticamente el rendimiento. Aunque la RMT puede mejorar el rendimiento, un alto porcentaje de equipo nuevo debe ser mantenido activamente por una fábrica. Eventualmente, el equipo se envejece y por tanto lo reciente del equipo va a declinar. Así pues, usar tales productores HP como modelo para identificar prácticas exitosas podría ser un negocio arriesgado (Maier and Schroeder, 2001).

Efectivamente, invertir en ambos tipos de tecnología, de productos y de procesos, es fundamental hacia el HPM. Ambos enfoques, usados juntos, son más eficaces que cualquiera de ellos invertidos de manera aislado, ya que tienden a tener un efecto sinérgico. Maier y Schroeder (2001) dan un ejemplo de que la inversión de la tecnología de procesos permite a las fábricas obtener una parte del mercado. Sin embargo, una estrategia que se enfoca sólo en la tecnología de procesos puede no necesariamente llevar a un crecimiento continuo a largo plazo, al menos que el mercado crezca por fuerzas externas. La tecnología de productos y el desarrollo de nuevos mercados son las únicas causas para el crecimiento sostenible e internamente influenciado. Por ende, estos autores recomiendan que, para apoyar los procesos de crecimiento con tecnologías de nuevos productos, las fábricas deben mejorar la tecnología de procesos.

Por tanto, basado en el análisis del HPM sobre las fábricas, en un camino donde las prácticas de producción llevan al alto rendimiento y competitividad, las fábricas deben tener ambas perspectivas simultáneamente, de una manera amplia y dinámica. El programa de tecnología ayuda a definir este camino, donde cada fábrica debe seguirlo conforme su propia contingencia, debido a que simplemente no es suficiente ser el líder en un área de la tecnología u otra.

\subsection{RMS Y LEAN}

Aunque se puede decir que el contexto reconfigurable encuentra algo de apoyo en algunos de los elementos esenciales de la producción lean, o que algo de dicho contexto puede de alguna manera derivarse del lean, existen algunas claras divisiones entre los dos.

La producción lean es vista comúnmente como una simple mejora de los métodos de producción en masa. El RMS, por su lado, tiende a distinguirse de la producción en masa, debido a que puede permitir la fabricación de productos altamente personalizados, cuando el cliente los necesita en la cantidad requerida. Asimismo, mientras lean tiene un modelo de producción capaz de operar eficazmente cuando hay condiciones de mercado estables, el RMS parece estar ajustado a enfrentar situaciones turbulentas por sus características de grado de reacción.

Finalmente, las dimensiones de rendimiento dentro del lean, alta eficiencia y productividad, están por encima del de alta grado de reacción (Yusuf et al., 1999). Sin embargo, como se explica en el subepígrafe 3.1, ambos, eficiencia y grado de reacción, son de igual importancia en el RMS. Así pues, lean puede ser mejor para las corridas largas que el RMS, ya que no es tan reaccionario y adaptable a los asuntos diarios, o a las necesidades de industrializar un nuevo producto con poca antelación, o a adap- 
tarse a nueva demanda. Para ello, el desafío es aún más agilidad, que puede llevar a procesos reconfigurables que el RMS parece proveer. La Tabla 1 ayuda a ilustrar algunas de las diferencias entre lo qué es una fábrica lean actual y lo qué podría ser una fábrica reconfigurable futura.

Por tanto, como se puede apreciar en la tabla 1, las diferencias de contexto y prácticas entre fábricas lean y

Volumen I

$\mathbf{N}^{\circ} 2$

2010 reconfigurables no son tan grandes si se gestionan apropiadamente. Además de los paradigmas de contingencia, de integración, de mejora continua y de dinamismo, para que una fábrica lean (sin equipo reconfigurable) pueda evolucionar hacia una reconfigurable, debe tomar en consideración los siguientes puntos vitales:

- Menos énfasis en procesos de alta automatización al tener más equipos polivalentes y reconfigurables: RMS.

- Más cantidad de equipo liviano, y más líneas paralelas en lugar de monolíticas para reconfigurar el diseño más fácilmente: RMS:

- Flexibilidad de principio a fin con tiempos de cambios muy reducidos de los procesos principales para obtener una alta velocidad del grado de reacción: RMS.

- Creación de una cultura alrededor de procesos alternativos y no de productos.

\subsection{RMS ¿nueva práctica, nueva máquina, o nuevo programa?}

RMS juega un papel importante en el futuro cercano según los puntos de vista actuales tales como programas de tecnología y producción lean. Sin embrago, como se ha mostrado, en un camino HPM es muy importante determinar si el RMS realmente significa una nueva práctica de producción, o algo más holístico como un programa/sistema/iniciativa, o solamente una nueva máquina disponible a toda fábrica. Como se ve en la sección 3.1, una razón para ello es que si el RMS es una nueva iniciativa, una fábrica cualquiera debe poseer algún tipo especial de destreza o capacidad al adoptarla antes que lo hagan sus competidores. Además, después de la primera adopción de la iniciativa por una fábrica de Clase Mundial, los competidores se ven forzados a imitar, si no se quieren quedar atrás en su rendimiento y competitividad. Dado que las iniciativas en general son sistémicas, por tanto muy complejas, no pueden simplemente ser adquiridas en la misma forma que una fábrica adquiriría una nueva máquina. El proceso de emular una iniciativa es complejo y sin ninguna garantía de éxito, ya que demandan esfuerzos complejos y periodos de mucho tiempo para su implementación. Así pues, las diferencias entra las capacidades de cada fábrica puede ser decisivas en determinar el éxito o el fracaso de la intervención hecha. Es casi imposible para la mayoría de las fábricas mantener los costos de invertir simultáneamente en muchas áreas, por lo que deben primero decidir cuáles son sus prioridades y después escoger (Filippini et al., 2001). Igualmente, las fábricas tampoco deben descartar al RMS, cuando ya esté al principio de su ciclo y que tan sólo empiece a ser introducido como una iniciativa innovadora. 


\begin{tabular}{|c|c|c|}
\hline Fábrica lean & Posible fábrica reconfigurable & \multirow{6}{*}{$\begin{array}{l}\text { Sistema de } \\
\text { Manufactura } \\
\text { Reconfigurable y } \\
\text { Competitividad } \\
\text { Industrial } \\
\end{array}$} \\
\hline Muy estable y un mercado grande. & $\begin{array}{l}\text { Mercado inestable, incierto, impredecible y competiti- } \\
\text { vo. }\end{array}$ & \\
\hline $\begin{array}{l}\text { Diseño fijo y optimizado para flujos y corri- } \\
\text { das. }\end{array}$ & $\begin{array}{l}\text { Diseño fácilmente reconfigurable, optimizado en visibi- } \\
\text { lidad. }\end{array}$ & \\
\hline Producción en tamaño de lote pequeño. & Todos los niveles de producción. & \\
\hline $\begin{array}{l}\text { Tecnología de uso general con partes de } \\
\text { automatización: menos equipos relativa- } \\
\text { mente flexibles, poca polivalencia y produc- } \\
\text { ción media-alta (incluido el FMS). }\end{array}$ & $\begin{array}{l}\text { Tecnología reconfigurable y fiable: más equipos con alta } \\
\text { polivalencia, menor nivel de producción y más líneas } \\
\text { paralelas. }\end{array}$ & \\
\hline $\begin{array}{l}\text { Operaciones balanceadas y sincronizadas. } \\
\text { Mejora continua (Kaizen). Tiempos reduci- } \\
\text { dos de preparación y cambio de equipo. } \\
\text { Tiempos de ciclo reducido. Eliminación de } \\
\text { residuo. Flujo de tamaño de lote. Células de }\end{array}$ & $\begin{array}{l}\text { Procesos de manufactura reconfigurable. No automati- } \\
\text { zación permanente de manufactura. Uso de TIC. Mejora } \\
\text { continua y radical. Tiempos reducidos de preparación y } \\
\text { cambio de equipo. Tiempos de ciclo reducido. Econo- } \\
\text { mías de escala y de gama. }\end{array}$ & \\
\hline $\begin{array}{l}\text { Manipulación automatizada para evitar per- } \\
\text { sonal. }\end{array}$ & Manipulación diseñada para hacer reconfiguración. & \\
\hline Visión de listo para ensamblar. & Objetivo permanente de estar listo para manufactura. & \\
\hline Calidad, productividad y flexibilidad. & $\begin{array}{l}\text { Flexibilidad para cambios inesperados } \\
\text { (reconfigurabilidad). Alta velocidad de grado de reac- } \\
\text { ción. }\end{array}$ & \\
\hline $\begin{array}{l}\text { Componente de estandarización para poder } \\
\text { estandarizar procesos. }\end{array}$ & Alternativas de procesos para tener reconfigurabilidad. & \\
\hline $\begin{array}{l}\text { Control de procesos estadísticos (Statistics } \\
\text { process control, SPC) de productos a proce- } \\
\text { sos. }\end{array}$ & $\begin{array}{l}\text { Diagnosticabilidad de procesos para capacidad variable, } \\
\text { funcionalidad y convertibilidad de familia de productos. }\end{array}$ & Pág 109 \\
\hline Diseño restrictivo de productos. & $\begin{array}{l}\text { Sistema abierto para futuros productos y cambios de } \\
\text { productos. }\end{array}$ & \\
\hline Conjunto de productos manejables. & Soluciones de producto basado en el valor. & \\
\hline Integración de procesos de automatización. & $\begin{array}{l}\text { Integración de procesos semi-automatizados o flexible- } \\
\text { mente automatizados pero reconfigurables. }\end{array}$ & \\
\hline
\end{tabular}

Tabla 1. Fábrica lean vs fábrica reconfigurable (Ajustado de Sahin, 2000; SISTEPLANT, 2003; Avella y Vásquez, 2005) 
En esta agenda, se ha explicado que el RMS se enfoca en nuevas maneras de diseñar y operar sistemas de producción por medio del cual las fábricas alcanzaran alto grado de reacción que sea costo eficaz (Koren et al., 1999). Por tanto, las prácticas reconfigurables futuras ciertamente prometen desarrollar un valor competitivo. Con todo, para que el RMS tenga realmente valor competitivo como un sistema de manufactura, debe estar apoyada por un fundamento, que no se limite al recurso mismo (RMT), sino que se extienda también a las prácticas de producción (sistema reconfigurable) como un todo ajustado en la fábrica. Además, debe tomar en consideración la naturaleza multidimensional del rendimiento y la contingencia de la fábrica involucrada en adoptar e implementar esta iniciativa (Schroeder y Flynn, 2001). En otras palabras: ir más allá del argumento de la universalidad de la "mejor práctica".

\section{CONCLUSIONES Y FUTURA INVESTIGACIÓN}

Aunque el RMS no está aún operativo y debe tenerse precaución cuando se generalice como una futura "mejor practica" en producción, parece haber espacio para el RMS en algunos contextos industriales, donde pueda jugar un papel importante en el alto rendimiento y la competitividad.

La literatura sobre el RMS establece una comparación entre éste con el FMS, así como con el lean. Por su lado, el HPM confirma la importancia de ambos, lean and FMS, sobre la competitividad y el alto rendimiento. Así pues, el RMS parece tener el potencial de ser una de las iniciativas más eficaces para mejorar el rendimiento, pero hay dos importantes asuntos que considerar al implementarlo en el contexto adecuado: 1) para que este en el camino correcto al HPM debe vincularse a otras prácticas en una fábrica; y 2) no es la solución completa que satisfaga todas, o aun la mayoría de, las dimensiones del rendimiento de producción, como para simplemente sustituir por completo las prácticas y sistemas actuales de producción.

En términos prácticos, puede decirse que existen muchos prototipos de RMSs ya desarrollados, la mayoría de los cuales son sistemas a nivel de máquina, pero la literatura especializada no muestra ningún intento especifico hecho para vincular operativamente un RMS a otras prácticas de producción. Por tanto, basados en las investigaciones y observaciones del presente artículo, se pueden identificar algunas direcciones futuras: 1 ) establecer un marco de referencia, que provea una taxonomía del HPM sobre el lean y la tecnología para ilustrar no sólo los puntos en común sino las diferencias en relación al RMS; y 2) poner un énfasis sobre la contingencia y el vínculo para poner en perspectiva los entornos y prácticas actuales de producción donde el RMS puede ajustarse.

\section{AgRADECIMIENTOS}

El presente estudio ha sido hecho en el marco del Ministerio de Ciencia e Innovación, proyectos DPI-200804788, DPI-2006-05531 and DPI-2009-11148, y en el marco del proyecto de la Junta de Andalucía P08-SEJ03841). Los autores desean agradecer al gobierno de España y al de Andalucía por sus apoyos parciales. 


\section{REFERENCIAS}

Amico, M., Asl, F., Pasek, Z. y Perrone, G. (2003). Real options: an application to RMS investment evaluation, proceeding of CIRP 2nd Conference on Reconfigurable Manufacturing, Ann Arbor MI, USA.

Avella, L. y Vázquez, D. (2005): Es la fabricación ágil un nuevo modelo de producción, Universia Business Review, Segundo Trimestre, 94-107.

Sistema de Manufactura Reconfigurable y Competitividad Industrial

Bi, Z.M., Lang, S.Y.T., Shen, W. yWang L. (2007): Reconfigurable manufacturing systems: the state of the art International Journal of Production Research, 46 (4), 967-992.

Cua, K., McKone-Sweet, K. y Schroeder, R. (2006). Improving Performance through an Integrated Manufacturing Program. The Quality Management Journal, 13 ( 3), 45.

EIMaraghy, H.A. (2006). Flexible and reconfigurable manufacturing systems paradigms. International Journal of Flexible Manufacturing Systems, 17, 261-276.

Filippini R., Vinelli A. y Voss C. (2001). Paths of Improvement in Operations. In Schroeder, R.G. y Flynn, B.B. (Eds.), High Performance Manufacturing - Global Perspectives., NEW YORK: John Wiley \& Sons (USA), 19-40.

Filippini, R., Forza C. y Vinelli A. (1998). Sequences of operational improvements: some empirical evidence, International Journal of Operations \& Production Management, 18 (2), 195-207.

Flynn, B.B., Schroeder, R.G., Flynn, E.J., Sakakibara, S. y Bates, K.A. (1997). World-class manufacturing project: overview and selected results, International Journal of Operations \& Production Management, 17 (7), 671-85.

Goldman, S.L., Nagel, R.N. y Preiss, K. (1995). Agile Competitors and Virtual Organizations: Strategies for Enriching the Customer, New York: Van Nostrand Reinhold.

Hayes, R.H.y Wheelwright, S.C. (1984). Restoring Our Competitive Edge: Competing through Manufacturing, John Wiley \& Sons, New York, NY.

Ketokivi, M. y Schroeder, R.G. (2004). “Manufacturing Practices, Strategic Fit and Performance: A Routine-Based View."International Journal of Operations \& Production Management, 24(2), 171-192. 
Liles, D.H. yHuff, B.L. (1990). A computer based production scheduling architecture suitable for driving a reconfigurable manufacturing next term system. Computers \& Industrial Engineering 19 (1-4), 1-5.

Maier, F. y Schroeder, R. (2001). Competitive Product and Process Technology. In: Roger G. Schroeder y Barbara B. Flynn (Eds.),High Performance Manufacturing, Global Perspectives, John Wiley \& Sons, Inc.

Volumen I

$\mathbf{N}^{\circ} 2$

2010 New York et al., 93-114.

Maier, F.H. (1997). Competitiveness in Manufacturing as Influenced by Technology-Some Insights from the Research Project: World Class Manufacturing. In Yaman Barlas, Vedat G. Diker, y Segkin Polat (Eds.), Systems Approach to Learning and Education into the 21st Century, 2, 667-670. Istanbul.

Mehrabi, M. G., Ulsoy, A.G. y Koren, Y. (2000a). Reconfigurable manufacturing systems and their enabling technologies. International Journal of Manufacturing Technology and Management, 1 (1), 113-130.

Mehrabi, M. G., Ulsoy, A.G. y Koren, Y. (2000b). Reconfigurable manufacturing systems: key to future manufacturing. Journal of Intelligent Manufacturing, 11, 403-419.

National Research Council (NRC) (Commission on Engineering and Technical Systems) (1998).Visionary Manufacturing Challenger for 2020, National Academy Press.

Next-Generation Manufacturing (NGM) Project (1997). Next-Generation Manufacturing: A Framework for Action, Agility Forum, Leaders for Manufacturing, and Technologies Enabling Agile Manufacturing, Bethlehem, PA.

Ortega, C.H. (2008). Producción de Alto Rendimiento en Honduras: Primer Acercamiento, Economía Política (Ahora Economía y Administración), 35, 57-74.

Ortega, C.H. (2009). Vinculo Estrategia de Operaciones-Tecnología en la Industria Hondureña: Ajuste de Selección, Economía Política (Ahora Economía y Administración), 48 (2), 133-148.

Pham, D.T., Eldukhri, E.E., Peat, B., Sctehi, R., Soroka, A., Packianather, M.S., Thomas, A. yDimov, S. (2004). "Innovativeproduction machines and systems," 2nd IEEE International Conference on Industrial Informatics, Berlin. Germany.

Sahin, F. (2000): Manufacturing competitiveness: Different systems to achieve the same results, Production and Inventory Management Journal, 41 (1) 56-65.

Schonberger, R.J. (1986).World-Class Manufacturing: The Lessons of Simplicity Applied, The Free Press, New York, NY.

Koren, Y., Heisel, U., Jovane, F., Moriwaki, T., Pritchow, G., Van Brussel, H. y Ulsoy, A.G. (1999). "Reconfigurable Manufacturing Systems," CIRP Annals, 48 (2), 527-540.

Schonberger, R.J. (2001).Let's Fix It! Overcoming the Crisis in Manufacturing: How the World's Leading Manufacturers Were Seduced by Prosperity and Lost Their Way, The Free Press, New York, NY 
Schroeder, R. G. y Flynn, B. B. (Eds.) (2001). High Performance Manufacturing: Global Perspectives, New York: John Wiley and Sons.

Schroeder, R., Bates K. y Junttila, M. (2002). A resource-based view of manufacturing strategy and the relationship to manufacturing performance, Strategic Management Journal, 23, 105-117.

SISTEPLANT (2003): Del Lean al Agile Manufacturing: La fabricación del próximo futuro, SISTEPLANT. Disponible: http://www.sisteplant.com (acceso 10 septiembre 2010).

Stecke, K.E. (2005). Flexibility is the future of reconfigurability. Paradigms of Manufacturing-A PanelDiscussion, $3^{\text {rd }}$ Conference on Reconfigurable Manufacturing, Ann Arbor, Michigan, USA.

Voss, C.A. y Blackmon, K. (1996). The impact of national and parent company origin on world-class manufacturing, Journal of Operations \& Production Management, 16 (11).

Yusuf, Y.Y., Sahardi, M. y Gunasekaran, A. (1999). Agile manufacturing: The drivers, concepts and attributes, International Journal of Production, Economics, 62 (1/2), 33-43. 